\title{
Structural Analysis and dynamics Simulation of Orthodontic Archwire Bending Robot
}

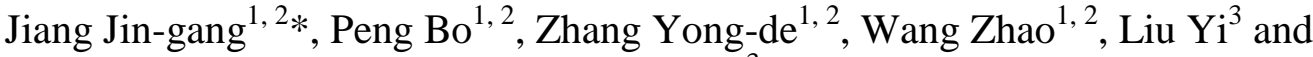 \\ Wen Fu-jia ${ }^{3}$ \\ ${ }^{1}$ Intelligent Machine Institute, Harbin University of Science and Technology, \\ Heilongjiang Harbin 150080, China \\ ${ }^{2}$ Robotics \& its Engineering Research Center, Harbin University of Science and \\ Technology, Harbin 150080, China \\ ${ }^{3}$ Peking University School of Stomatology, Beijing, 100081, China \\ jiangjingang@hrbust.edu.cn
}

\begin{abstract}
The structural dynamics in archwire-bending process is critical to the orthodontic archwire bending robot. In this paper, structure of orthodontic archwire bending robot is analyzed. Transient dynamic of orthodontic archwire bending is analyzed using the transient dynamic module of ANSYS/Workbench software. Stress curve and distribution of orthodontic archwire, fixed die, rotation die and supported end of orthodontic archwire in the process of orthodontic archwire bending is obtained. The structural dynamics simulation lays the foundation for the structural design of orthodontic archwire bending robot.
\end{abstract}

Keywords: structural dynamics simulation, structure analysis, orthodontic archwire bending robot

\section{Introduction}

Traditionally, orthodontic archwire is bent by orthodontist by manual operation[1]. However, this process is boring and inefficient. A robotic apparatus was introduced to free orthodontist from hard work [2]. The archwire bending process plays a major role in orthodontic treatment [3-4]. In the bending process, after release of the load by loosening the fixture, the archwire tries to return to its original shape because of the elastic stresses[5]. Structural dynamics of orthodontic archwire bending robot is an important factor in tooling design and obtaining the desired configuration of archwire. So study on structural dynamics of orthodontic archwire bending robot in archwire-bending process is a key issue [6-8]. Consequently, it is important and essential to study the structural dynamics of orthodontic archwire bending robot.

\section{Structure of Orthodontic Archwire Bending Robot}

Orthodontic archwire bending robot, which is consistent of archwire feeder, archwire bending structure, archwire support structure, fixed die feeder and the base, is as shown in Figure 1. Fixed die of fixed die feeder locates at the slipway. Up and down movement of fixed die is realized by the slipway. The rotation movement of rotation die revolving around the fixed die is realized by archwire bending structure, and archwire bending structure can adjust the position and posture of rotation die in order to ensure the relative position of rotation die and fixed die, and thus to realize the archwire bending. In order to prevent the warping deformation of orthodontic archwire, archwire support structure is designed. 


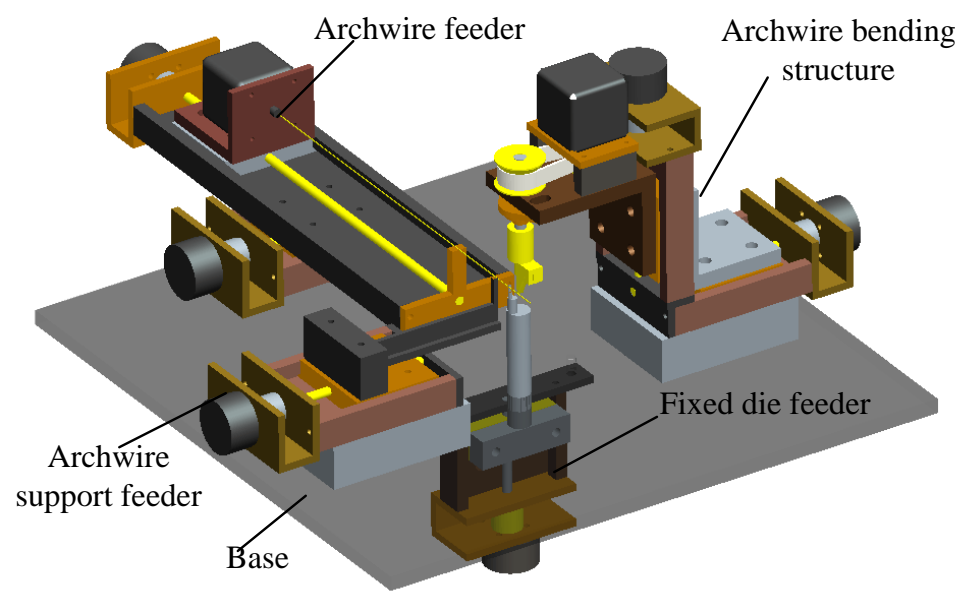

Figure 1. Structure of the Archwire Bending Robot

\section{Transient Dynamic Analysis of Orthodontic Archwire Bending Robot}

In the process of orthodontic archwire bending, the rotation die turns the orthodontic archwire to rotate around the fixed die. Thus the rotation die, orthodontic archwire and fixed die will distort in the interactive forces of three objects. So it is critical to analyze the transient dynamic analysis of archwire bending. Transient dynamic of orthodontic archwire bending is analyzed using the transient dynamic module of ANSYS/Workbench software.

\subsection{Simplification of Simulation Model}

In order to simplify the analytical calculation and improve the simulation accuracy, the simulation model of orthodontic archwire bending is simplified and established using the ANSYS/Workbench software. Simplification simulation model is as shown in Figure 1.

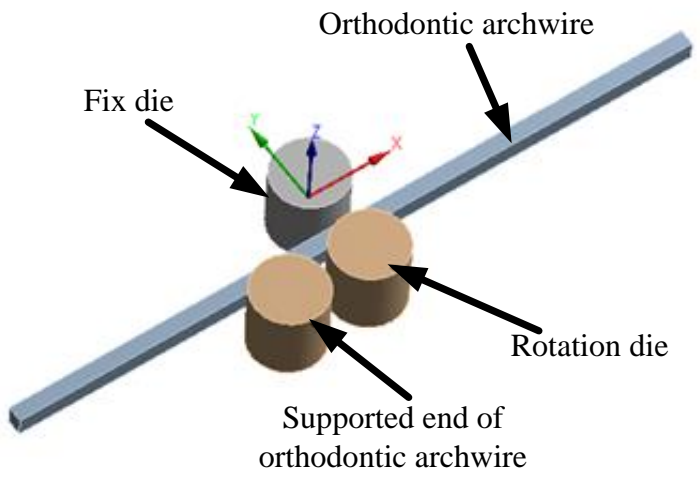

Figure 1. Simplification Simulation Model of Orthodontic Archwire Bending

\subsection{Setting of Material Property}

The plastic material, which is multi-linear isotropic material, is used in the simulation analysis. And according to the stress-strain curve of the Nickel titanium arch wire, the property of material is added to the simplification simulation model. The stress-strain curve of nickel titanium archwire is as shown in Figure 2. 


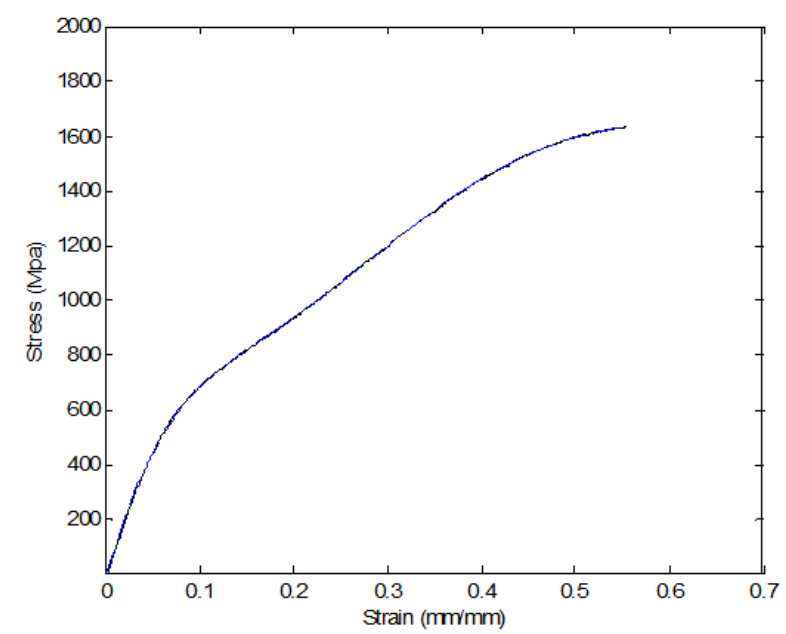

Figure 2. Stress-strain Curve of Nickel Titanium Archwire

\subsection{Mesh generation}

It has high quality of grid in the process of solution, so the grid size is set $0.2 \mathrm{~mm}$.

\subsection{Checking out of the Contact}

After the setting of the contact, the contact is solved. And then the contact relationship is checked out to judge whether the analysis condition is satisfied or not. From the result of analysis of mode contact, the maximum clearance between rotation die and orthodontic archwire is $1.2697 \mathrm{e}-4 \mathrm{~mm}$, the maximum clearance between fixed die and orthodontic archwire also is $1.2697 \mathrm{e}-4 \mathrm{~mm}$, the maximum clearance between the supported end and orthodontic archwire is $9.2183 \mathrm{e}-5 \mathrm{~mm}$. So the results meet the requirement of simulation analysis.

\subsection{Parameter Setting of the Analysis}

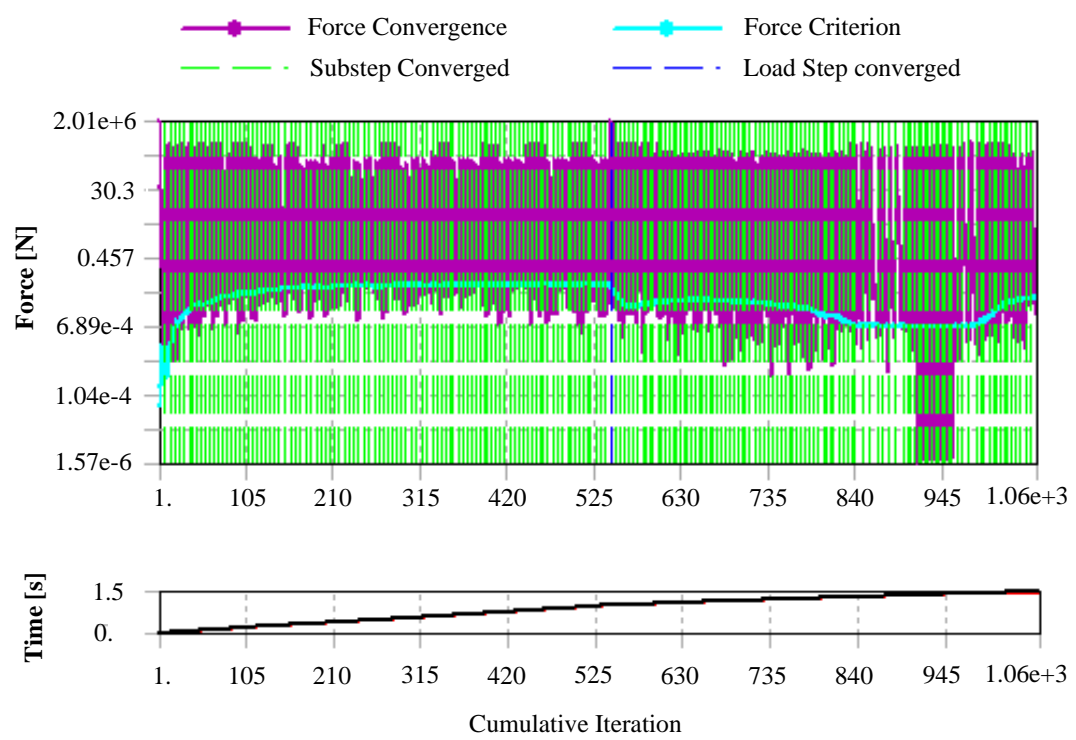

Figure 3. Convergence of Force 
The analysis, which continues $1.5 \mathrm{~s}$, is two steps. The first step continues $1 \mathrm{~s}$, and the bending action of orthodontic archwire is completed when the rotation die turns $90^{\circ}$. The second step continues $0.5 \mathrm{~s}$, and the return motion of rotation die is completed when the rotation die turns $90^{\circ}$ reversely. The convergence of the stress is checked out in the process of solving. If the simulation is failed, the analysis of parameter setting is adjusted again is as shown in Figure 3. From the convergence of force, we can see that all the convergence points fall under the convergence criterion, and it has solution.

\section{Analysis of the Results}

Stress curve of orthodontic archwire in the process of orthodontic archwire bending is as shown in Figure 4. From the Figure 4, we can see that the orthodontic archwire is in the range of elastic deformation at the beginning, and the stress increases with the time. The stress is to $500 \mathrm{Mpa}$ when time is about $0.25 \mathrm{~s}$, and orthodontic archwire access to the area of elastic deformation. This is accorded with property of material. Then the stress continues to increase, and the stress reaches the maximum value of $830.95 \mathrm{Mpa}$ when it is about $1 \mathrm{~s}$. After 1s, because the rotation die turns reversely, the archwire stress is reduced and the rebound takes place. And it has no change about $1.25 \mathrm{~s}$ to $1.4 \mathrm{~s}$. The result indicates that it has no mutual interaction between orthodontic archwire and rotation die, so it only exists residual stress. The deformation is accord with requirements. The stress of orthodontic archwire increases when it is about $1.4 \mathrm{~s}$ to $1.5 \mathrm{~s}$, this result indicates that it has mutual interaction between the archwire and rotation die. The reason is that the deformation of archwire in bending initial position is irregular. When the rotation die returns to the initial position, mutual interaction takes place between the rotation die and deformation archwire. Normal stress distribution of the orthodontic archwire at $1 \mathrm{~s}$ is as shown in Figure 5. Residual stress distribution of the orthodontic archwire at $1.5 \mathrm{~s}$ is as shown in Figure 6.

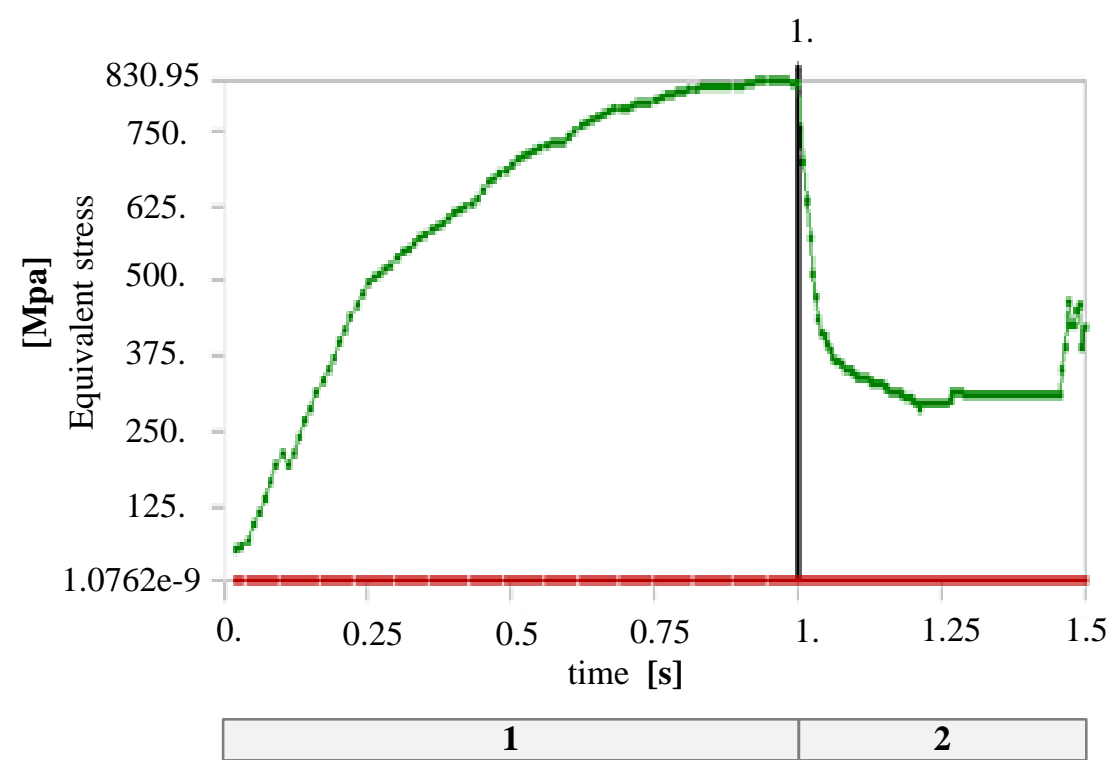

Figure 4. Stress Curve of Orthodontic Archwire in the Process of Orthodontic Archwire Bending 


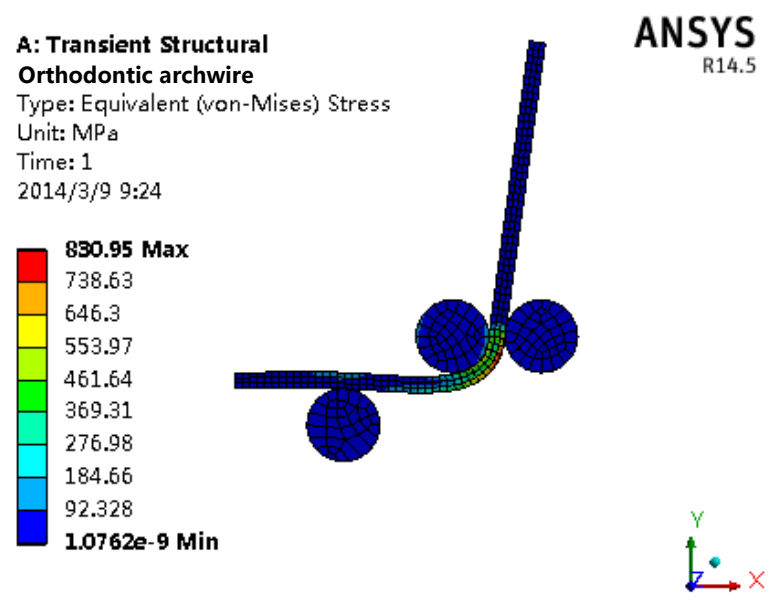

Figure 5. Normal Stress Distribution of the Orthodontic Archwire at 1s

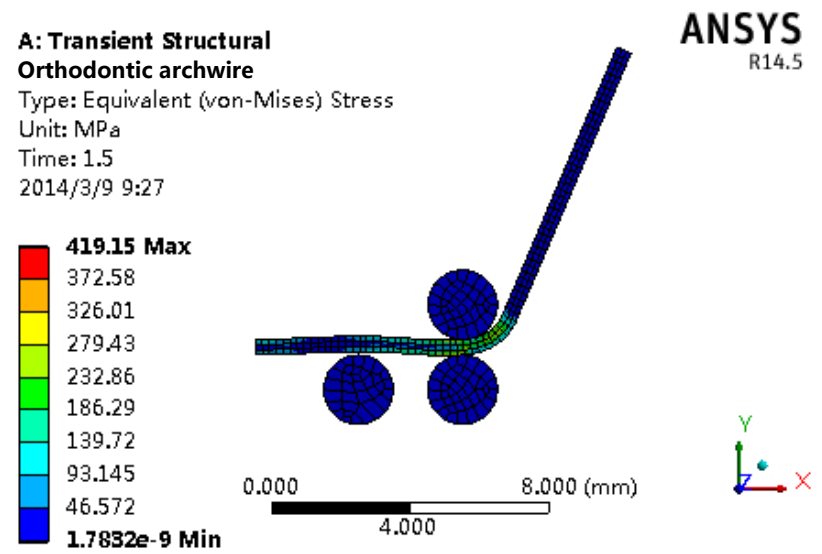

Figure 6. Residual Stress Distribution of the Orthodontic Archwire at 1.5s

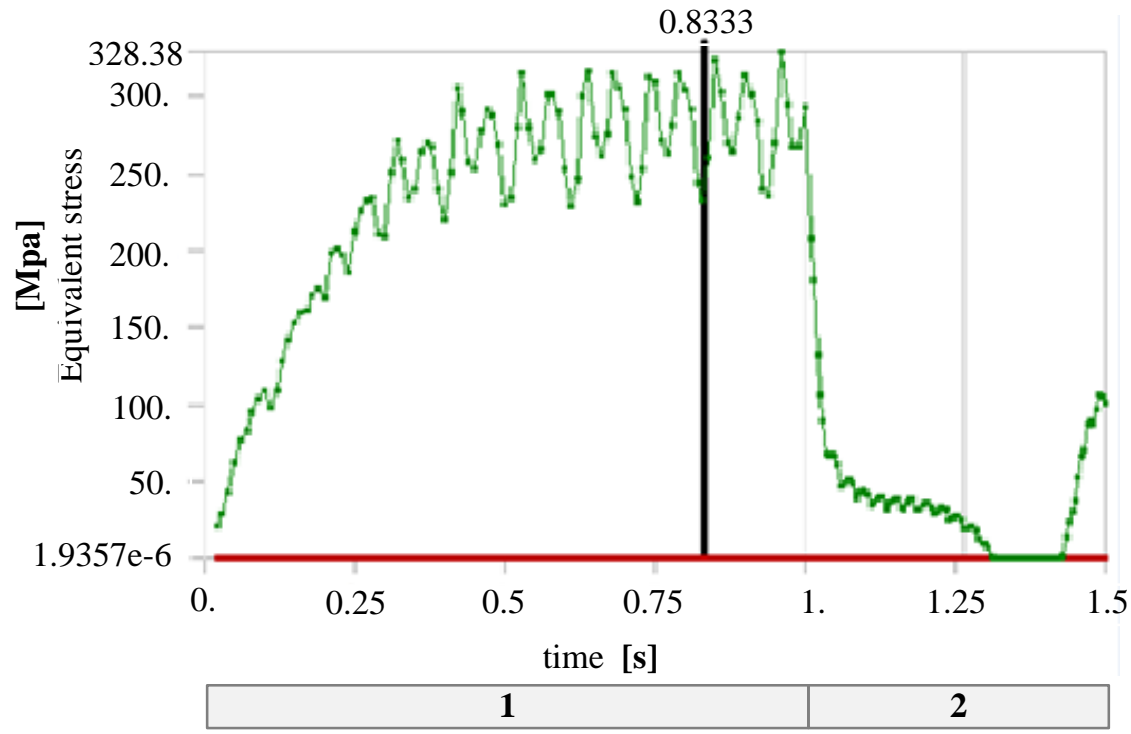

Figure 7. Stress Curve of Rotation Die in the Process of Orthodontic Archwire Bending

The stress curve of rotation die in the process of orthodontic archwire bending is as shown in Figure 7. The stress distribution of rotation die is as shown in Figure 8. From the 
Figure 4 , we can see that the maximum value of rotation die is $328.38 \mathrm{Mpa}$. It is close to the yield limit of $375 \mathrm{Mpa}$, and its fluctuation is larger than before. In order to ensure the strength, the rotation die can be heat-treated to improve the allowable stress of surface and its overall strength and stiffness.

The stress curve of fixed die in the process of orthodontic archwire bending is as shown in Figure 9. The stress distribution of fixed die is as shown in Figure 10. From the Figure 9 , we can see that the maximum value of rotation die is $473.5 \mathrm{Mpa}$. It exceeds the yield limit of $375 \mathrm{Mpa}$, and its fluctuation is large.

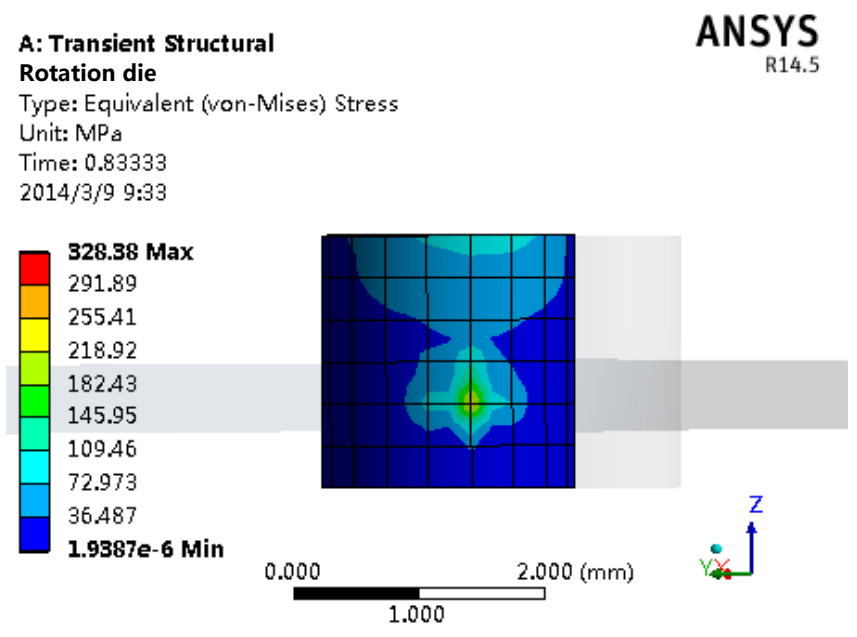

Figure 8. Stress Distribution of Rotation Die

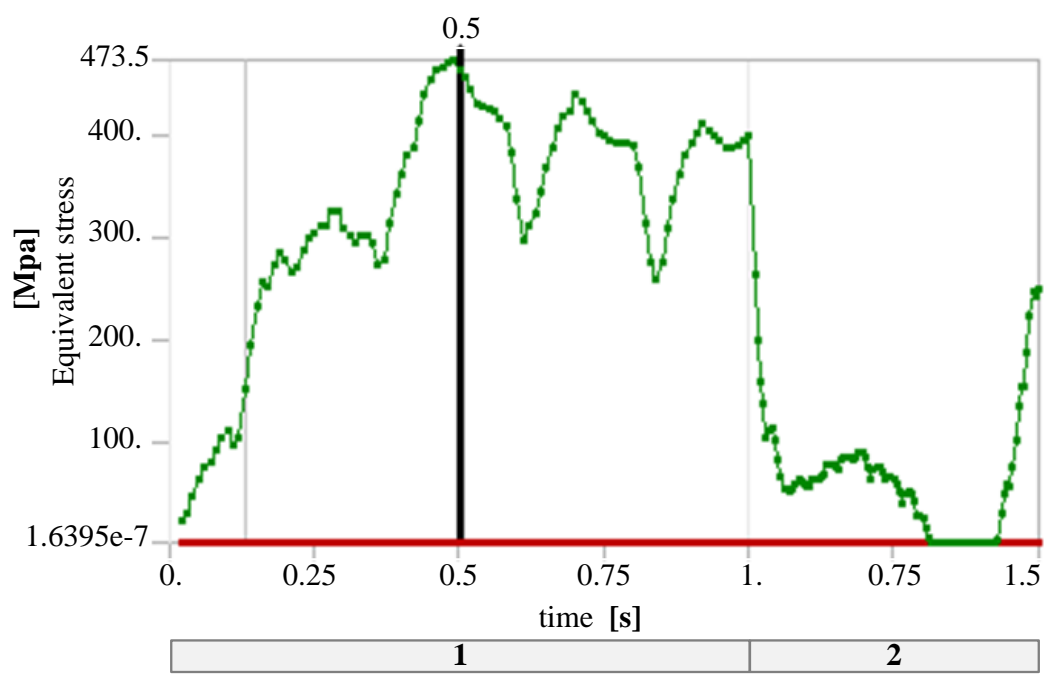

Figure 9. Stress Curve of Fixed Die in the Process of Orthodontic Archwire Bending 

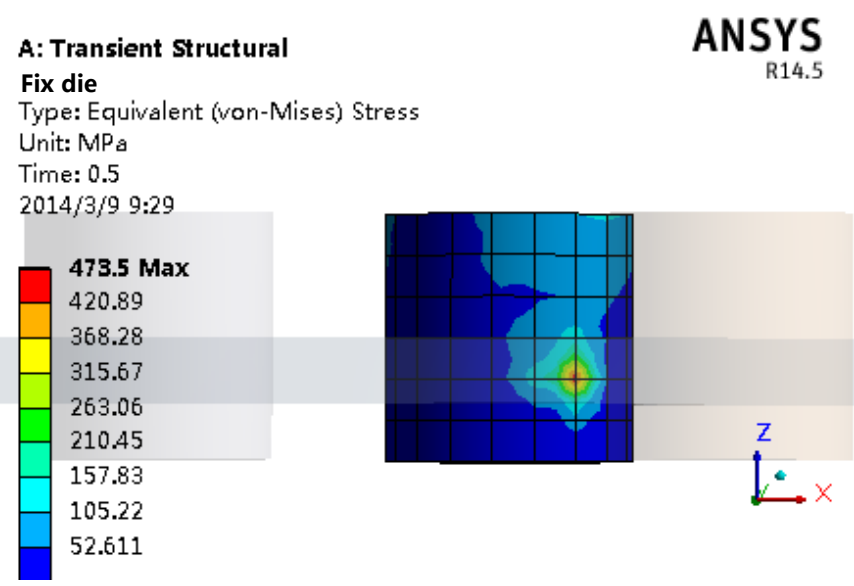

Figure 10. Stress Distribution of Fixed Die

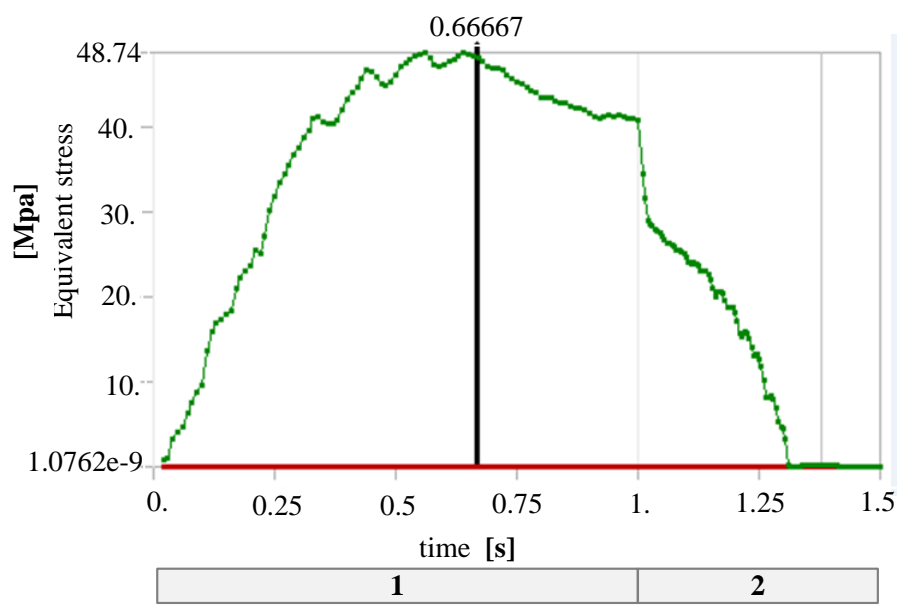

Figure 11. Stress Curve of Supported End of Orthodontic Archwire in the Process of Orthodontic Archwire Bending

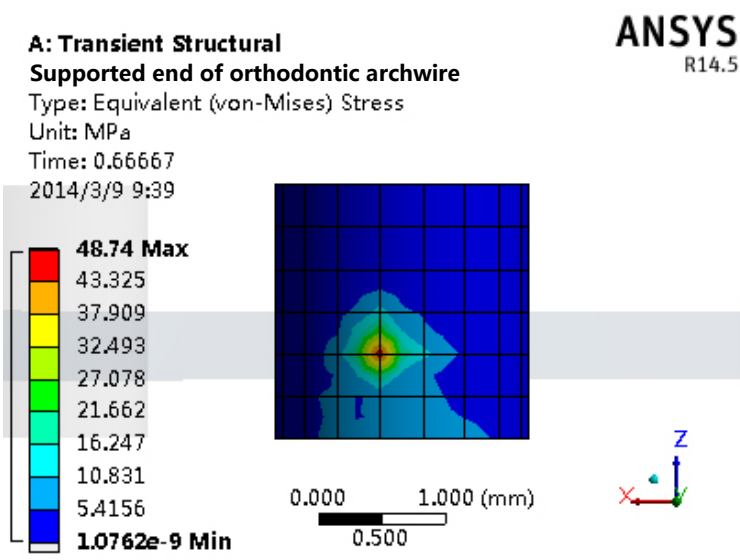

Figure 12. Stress Distribution of Supported End of Orthodontic Archwire

The stress curve of supported end of orthodontic archwire in the process of orthodontic archwire bending is as shown in Figure 11. The stress distribution of supported end of orthodontic archwire is as shown in Figure 12. From the Figure 11, we can see that the maximum value of supported end of orthodontic archwire is $48.74 \mathrm{Mpa}$. And it is accord with requirements. 


\section{Conclusions}

The structural dynamics in archwire-bending process is critical to the orthodontic archwire bending robot.

(1) Structure of orthodontic archwire bending robot is analyzed. Transient dynamic of orthodontic archwire bending is analyzed using the transient dynamic module of ANSYS/Workbench software.

(2) Stress curve and distribution of orthodontic archwire, fixed die, rotation die and supported end of orthodontic archwire in the process of orthodontic archwire bending is obtained. The structural dynamics simulation lays the foundation for the structural design of orthodontic archwire bending robot.

\section{Acknowledgements}

This research was supported by the National Natural Science Foundation of China (Grant No. 51205093), the Heilongjiang Province Education Bureau Project (Grant No. 12541147), and the National High Technology Research and Development Program of China (Grant No. 2013AA040803).

\section{References}

[1] Zhang Yongde, Jia Yuxiang. The control of archwire bending robot based on MOTOMAN UP6[C]. BMEI 09, 2nd International Conference on Biomedical Engineering and Informatics, Tianjin, China, 2009: 1057-1061.

[2] Jiang Jin-gang, Zhang Yong-de, Jin Ming-liang, et al. Bending process analysis and structure design of orthodontic archwire bending robot[J]. International Journal of Smart Home, 2013, 7(5): 345-352.

[3] Zhang Yongde, Jiang Jixiong. Analysis and experimentation of the robotic system for archwire bending[J]. Applied Mechanics and Materials, 2012, 121-126: 3805-3809.

[4] S. Partowi, L. Keilig, S. Reimann, A. Jager, C. Bourauel. Experimental analysis of torque characteristics of orthodontic wires [J]. Journal of Orofacial Orthopedics-fortschritte Der Kieferorthopadie, 2010, 71(5): 362-372.

[5] Jiang Jin-gang, Zhang Yong-de, Wei Chun-ge, et al. A review on robot in prosthodontics and orthodontics[J]. Advances in Mechanical Engineering, 2015, 7(1): 198748.

[6] Zhang Yongde, Wei Chunge, Jiang Jingang, et al. Motion planning for archwire bending robot in orthodontic treatments[J]. International Journal of Control and Automation, 2014, 7(7): 287-297.

[7] Jiang Jingang, Zhang Yongde. Motion planning and synchronized control of the dental arch generator of the tooth-arrangement robot[J]. The International Journal of Medical Robotics and Computer Assisted Surgery, 2013, 9(1): 94-102.

[8] Jiang Jin-gang, Wang Zhao, Zhang Yong-de, Jiang Ji-xiong, Niu Suo-liang, Liu Yi. Study on springback properties of different orthodontic archwires in archwire bending process [J]. International Journal of Control and Automation, 2014, 7(12): 283-290. 